\title{
Exercise-Based Rehabilitation and Manual Therapy Compared With Exercise-Based Rehabilitation Alone in the Treatment of Chronic Ankle Instability: A Critically Appraised Topic
}

\author{
Bridget M. Walsh, Katherine A. Bain, Phillip A. Gribble, and Matthew C. Hoch
}

\begin{abstract}
Clinical Scenario: Patients with chronic ankle instability (CAI) commonly display lower levels of self-reported function and healthrelated quality of life. Several rehabilitation interventions, including manual therapy, have been investigated to help CAI patients overcome these deficits. However, it is unclear if the addition of manual therapy to exercise-based rehabilitation is more effective than exercise-based rehabilitation alone. Clinical Question: Does incorporating manual therapy with exercise-based rehabilitation improve patient-reported outcomes when compared with exercise-based rehabilitation alone? Summary of Key Findings: The literature was searched for articles that examined the difference in outcomes for patients with CAI between manual therapy with exercise-based rehabilitation and exercise-based rehabilitation alone. A total of 3 peer-reviewed randomized controlled trials were identified. Two articles demonstrated improved patient-reported outcome scores following the incorporation of manual therapy with exercise-based rehabilitation, whereas one study found no statistically significant differences between interventions. Clinical Bottom Line: The current evidence suggests that incorporating manual therapy in addition to exercised-based rehabilitation may improve patient-reported outcome scores in patients with CAI. Strength of Recommendation: In accordance with the Strength of Recommendation Taxonomy, the grade of A is recommended due to consistent evidence from high-quality studies.
\end{abstract}

Keywords: ankle sprain, evidence-based practice, patient reported outcomes, joint mobilization, balance training

\section{Clinical Scenario}

In physically active populations, lateral ankle sprains are the most commonly occurring lower-extremity musculoskeletal injury. ${ }^{1}$ After sustaining an acute ankle sprain, only $6.8 \%$ of patients receive formal physical therapy for their injury. ${ }^{2}$ Further perpetuating the problem, $74 \%$ of those who sustain a single lateral ankle sprain develop residual symptoms that may persist years after the original injury, ${ }^{3}$ which could lead to the development of chronic ankle instability (CAI). Chronic ankle instability is defined as a history of at least 1 significant ankle sprain, giving way, recurrent sprain, and/or feelings of instability. ${ }^{4}$ Those with CAI often exhibit lifelong complications that impact physical activity levels as well as their health-related quality of life (HRQOL). ${ }^{5}$ Therefore, it is important that clinicians are aware of best practice and most effective rehabilitation strategies that improve outcomes in this patient population.

Manual therapy techniques are often used to improve pain, range of motion, and tissue extensibility. For these reasons, manual therapy interventions are commonly implemented for patients with CAI. There is evidence to suggest the use of manual therapy, specifically joint mobilization techniques, can improve clinician-based outcomes, such as ankle dorsiflexion range of motion or dynamic postural control in patients with CAI. ${ }^{6,7}$ Despite the limited number of studies that have examined the effect of manual therapies on patient outcomes, the evidence is

Walsh, Bain, and Gribble are with the Department of Athletic Training and Clinical Nutrition, College of Health Sciences, University of Kentucky, Lexington, KY. Hoch is with the Department of Athletic Training and Clinical Nutrition, Sports Medicine Research Institute, College of Health Sciences, University of Kentucky, Lexington, KY. Hoch (Matt.Hoch@uky.edu) is corresponding author. supportive that talocrural joint mobilization techniques have moderate effects on patient-reported function. ${ }^{6,8}$ The benefits of joint mobilization on mechanical, sensorimotor, and patientreported function are evident when these interventions are examined in isolation for patients with CAI. However, the effects of manual therapies used in conjunction with exercise-based interventions is not as clear but is vital to understanding the cumulative effect of these rehabilitation strategies. There is currently limited evidence to support whether incorporating manual therapy in addition to exercise-based rehabilitation can improve patientreported function in those with CAI. Therefore, the purpose of this critically appraised topic (CAT) was to critically appraise and synthesize the available evidence that compared these intervention strategies in patients with CAI.

\section{Focused Clinical Question}

Does incorporating manual therapy with exercise-based rehabilitation result in greater improvements in patient-reported outcome (PRO) scores compared with exercise-based rehabilitation alone in patients with CAI?

\section{Search Strategy}

A computerized search was completed in July 2019. The search terms used were

- Patient/Client: chronic ankle instability OR ankle instability OR functional instability OR mechanical instability

- Intervention: manual therapy OR joint mobilization OR mobilization with movement

- Comparison: rehabilitation 
- Outcome: patient-assessed OR patient-report OR questionnaire OR self-report OR scale

\section{Sources of Evidence Searched}

- CINAHL with full text

- MEDLINE

- SPORTDiscus

\section{Inclusion Criteria}

- Studies published in the English language

- Studies published in the last 10 years

- Studies that included human subjects

- Studies that incorporated at least 1 PRO assessing HRQOL

- Studies classified as level 2 evidence or higher

- Studies that involved patients with CAI

- Studies that incorporated manual therapy in conjunction with exercise-based rehabilitation (eg, strength training, proprioceptive training, balance training)

- Studies that incorporated a control group that received exercise-based rehabilitation as an intervention without joint mobilization

\section{Exclusion Criteria}

- Studies that involved patients with an acute lateral ankle sprain

- Studies that incorporated only manual therapy as an intervention

- Studies that included a control group that did not receive exercise-based rehabilitation as an intervention

- Studies that did not include a PRO

\section{Evidence Quality Assessment}

Included studies were critically appraised with the Physiotherapy Evidence Database (PEDro) rating scale. The PEDro scale was selected because it is commonly used to assess the methodological quality of randomized controlled trials (RCTs) and other study designs used to compare rehabilitation interventions. All included articles were critically appraised independently by 2 reviewers (B.M.W. and K.A.B.). After independent review, the 2 authors reviewed the completed appraisals and reached an agreement about study quality. The highest possible score on the PEDro is 10 . Articles which scored at least a $60 \%$ on the PEDro were classified as high-quality studies, whereas studies below $60 \%$ were classified as limited-quality studies. The Strength of Recommendation Taxonomy was used to assess the grade of evidence and provide a strength of recommendation. ${ }^{8}$ In summary, a strength of recommendation of $\mathrm{A}$ is reserved for consistent (similar or at least coherent conclusions), high-quality patient-oriented evidence. A recommendation of B is assigned when the evidence is inconsistent or limitedquality patient-oriented evidence. Finally, a recommendation of $\mathrm{C}$ is assigned for evidence that is considered opinion, disease-oriented, or case series.

\section{Results of Search}

\section{Summary of Search, Best Evidence Appraised, and Key Findings}

- A literature search was conducted to identify peer-reviewed articles that investigated the effectiveness of incorporating manual therapy in addition to exercise-based rehabilitation on PROs.

- The search yielded a total of 28 possible studies. After review of the titles and abstracts, 25 studies were excluded as they did not meet the inclusion criteria for this CAT. Three RCTs met the inclusion criteria (Table 1). Characteristics of each study are shown in Table 2.

- One study ${ }^{9}$ involved 2 groups: a balance training group and a balance training plus combination of calf-stretching, ankle joint traction, anterior/posterior ankle joint mobilizations, and plantar massage group. Both groups received treatment 3 times a week for $\sim 20$ minutes over a 4-week training protocol. There were no statistically significant differences between groups in the posttest values.

- Another study ${ }^{10}$ involved 2 groups: a proprioceptive and strengthening exercise group and a proprioceptive and exercise group with additional manual therapy. Both groups received treatment twice a week over a 4-week period. There were significant improvements in visual analog scale scores and Cumberland Ankle Instability Tool scores in the group receiving manual therapy at posttesting.

- The third included study ${ }^{11}$ involved 3 groups: a calf muscle stretching group; a neuromuscular training group that received sham joint mobilization; and a neuromuscular training group that received intermittent traction, anteroposterior mobilizations to the talus, and distal tibiofibular mobilizations. Groups received treatment twice a week for 4 weeks. There were significant improvements in CAIT scores between the manual therapy group and the stretching group at posttesting.

\section{Results of Evidence Quality Assessment}

Of the included studies, critical appraisal scores ranged from 6 to 10 , indicating all 3 studies were categorized as high-quality evidence. Plaza-Manzano et al $^{10}$ achieved a full score of 10 . Neither Burcal et $\mathrm{al}^{9}$ nor Shih et $\mathrm{al}^{11}$ described any efforts to perform blinding of subjects, therapists, or assessors to reduce potential bias, decreasing their scores each by 3 points. In addition, Shih et $\mathrm{al}^{11}$ did not describe any efforts to conceal group allocation, decreasing the score by another point.

\section{Clinical Bottom Line}

The current evidence suggests that incorporating manual therapy in addition to exercise-based rehabilitation creates greater improvements

Table 1 Summary of Evidence

\begin{tabular}{|c|c|c|c|}
\hline Level of evidence & Study design & Number located & References \\
\hline 1 & Randomized controlled trial & 3 & 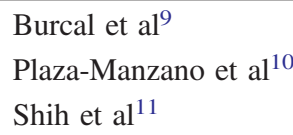 \\
\hline
\end{tabular}


Table 2 Summary of Articles

\begin{tabular}{|c|c|c|c|}
\hline & Plaza-Manzano et al ${ }^{10}$ & Burcal et al $^{9}$ & Shih et al ${ }^{11}$ \\
\hline Study design & Randomized controlled trial & Randomized controlled trial & Randomized controlled trial \\
\hline Participants & $\begin{array}{l}\text { A total of } 56 \text { participants were divided } \\
\text { into } 2 \text { intervention groups: experimental I } \\
\text { group }(\mathrm{n}=28 \text {, age }=24.1[2.4] \mathrm{y} \text {, height }= \\
176.7[8.9] \mathrm{m} \text {, weight }=74[10.8] \mathrm{kg}, \\
\mathrm{BMI}=23.6[1.8], 71.4 \% \text { male }) \text { and } \\
\text { experimental II group }(\mathrm{n}=28 \text {, age }=24.4 \\
{[2.4] \mathrm{y}, \text { height }=176.5[8.8] \mathrm{m} \text {; weight }=} \\
73.7[13.1] \mathrm{kg}, \mathrm{BMI}=23.0[1.4], 67.9 \%\end{array}$ & $\begin{array}{l}\text { A total of } 24 \text { participants were divided } \\
\text { into } 2 \text { intervention groups: BT group } \\
(\mathrm{n}=12, \text { age }=21.17[1.64] \mathrm{y} \text {, height }= \\
170.82[15.09] \mathrm{cm} \text {, mass }=73.04 \\
[24.76] \mathrm{kg}, 22.7 \% \text { male }) \text { and BT with } \\
\text { STARS group }(\mathrm{n}=12, \text { age }=21.42 \\
{[2.43] \mathrm{y}, \text { height }=168.80[10.85] \mathrm{cm} \text {, }} \\
\text { mass }=71.93[20.30] \mathrm{kg}, 16.7 \% \text { male })\end{array}$ & $\begin{array}{l}\text { A total of } 45 \text { participants were divided } \\
\text { into } 3 \text { groups: control group }(\mathrm{n}=15, \text { age }= \\
27.9[6.6] \mathrm{y}, \mathrm{BMI}=22.2[2.5], 46.7 \% \\
\text { male }) \text {, training group }(\mathrm{n}=15 ; \text { age }=26.9 \\
[5.8], \mathrm{BMI}=22.1[2.6], 46.7 \% \text { male }), \text { and } \\
\text { mobilization with training group } \\
(\mathrm{n}=15 ; \text { age }=26.5[4.8] \text { y, BMI }=23.3 \\
[3.2], 66.7 \% \text { male })\end{array}$ \\
\hline
\end{tabular}

Inclusion criteria Previous initial PFI ankle sprain graded I, II, or III at least 12 mo prior to the beginning of the study, recurrence of previous PFI ankle sprains, no history of sprain of the affected ankle in the last $3 \mathrm{mo}$, and recreational activity at least 3 times a week

Exclusion criteria

Surgical treatments, previous fractures in either lower-extremity, adjacent pathologies that disturbed the joint integrity or function and required at least $1 \mathrm{~d}$ of interrupted physical activity

Intervention investigated

Outcome measures

Main findings

Level of $1 b$

Experimental I group: proprioceptive and strengthening exercises over a 4-wk period, twice a week experimental II group: same proprioceptive and strengthening exercises as group I, plus additional manual therapy over the same 4-wk period, twice a week. The manual therapy intervention consisted of talocrural joint mobilizations in distraction, posteroanterior talocrural joint mobilization, anteroposterior talocrural joint mobilization, anteroposterior and posteroanterior distal tibiofibular joint mobilizations, and superficial peroneal nerve neurodynamic mobilization.

VAS pain scale, CAIT, PPT, active ankle range of motion, dynamometry strength

Experimental II group exhibited significant lower levels of pain and higher scores on the CAIT immediately after intervention as well as at a 1-mo followup. The experimental II group also exhibited both higher PPT values for all points and higher strength values for both flexion and extension. The experimental II group had increased ankle flexion and extension range of motion values compared with the experimental I group.
Physically active (at least $90 \mathrm{~min}$ of cardiovascular activity or equivalent per week), history of CAI (determined using AII, FAAM, and FAAM-S)

Known balance or vision problems, acute lower-extremity injuries, head injuries, chronic musculoskeletal conditions known to affect balance, history of ankle surgery

BT group: 4-wk supervised BT protocol with three, 20-min sessions per week

BTS group: the same BT protocol as BT group with a combination of calf stretching, ankle joint traction, anterior/ posterior ankle joint mobilizations, and plantar massage

SEBT, static single-limb stance trials, FAAM, FAAM-S

There were no significant group $\times$ time interactions found for any of the included outcome measures. All of the $90 \%$ confidence interval for betweengroup differences crossed 0 , indicating a lack of difference.
Between 20 and $50 \mathrm{y}$ old, history of at least 1 ankle sprain within the past year with residual symptoms or having repetitive ankle sprains within the past year with feelings of "giving way" within the past $3 \mathrm{mo}$, CAIT score $\leq 27$

History of fracture or surgery to the lower-extremity, injury to the lowerextremity within the last $3 \mathrm{mo}$, ankle sprain in the past $6 \mathrm{wk}$, pathological joint laxity (positive talar tilt or anterior drawer tests)

CG: calf muscle stretching instruction TG: 15-20 min of neuromuscular training combined with a 15 -min sham joint mobilization throughout a 4-wk period

MTG: 8 identical neuromuscular training sessions combined with joint mobilization during a 4-wk period. Mobilizations included intermittent traction in the longitudinal direction of the talocrural joint, grades III and IV AP mobilizations to the talus, and grades III and IV mobilizations to the distal tibiofibular joint

YBT, EMG muscle activation during YBT, ankle ROM, CAIT, GRS

There were significant improvements in CAIT scores in the MTG compared with the CG. There were significant improvements in both the MTG and the TG compared with the CG in GRS scores. There was also a significant improvement in dorsiflexion ROM in the MTG group compared with the $\mathrm{CG}$, whereas no significant improvement was noted for the TG. Only the MTG group showed significant improvements in the posterolateral reach of the YBT. Peroneus longus activation decreased significantly in the MTG compared with the CG and TG.

$1 \mathrm{~b}$

PEDro: 6 
Table 2 (continued)

\begin{tabular}{|c|c|c|c|}
\hline & Plaza-Manzano et al ${ }^{10}$ & Burcal et al ${ }^{9}$ & Shih et al ${ }^{11}$ \\
\hline Conclusion & $\begin{array}{l}\text { Incorporating manual therapy in addition } \\
\text { to proprioceptive training and strength- } \\
\text { ening in patients with CAI decreases } \\
\text { pain, increases self-reported function } \\
\text { scores, increases PPT values, and } \\
\text { increases ROM. }\end{array}$ & $\begin{array}{l}\text { BT combined with STARS is no more } \\
\text { effective than BT alone. }\end{array}$ & $\begin{array}{l}\text { Incorporating mobilization in addition to } \\
\text { training improves not only scores on the } \\
\text { CAIT and GRS but also improves dor- } \\
\text { siflexion ROM, posterolateral reach, and } \\
\text { posterolateral activation. }\end{array}$ \\
\hline
\end{tabular}

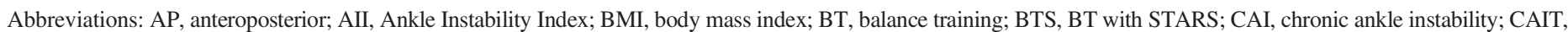

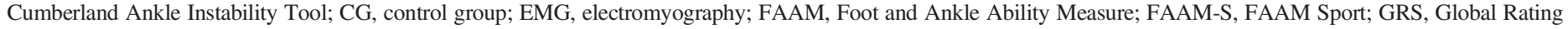

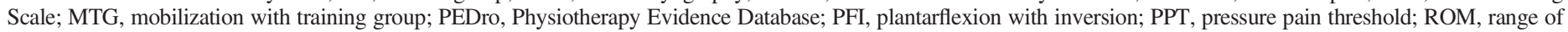

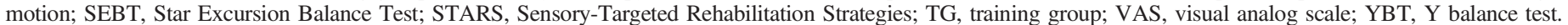

in PRO scores compared with exercise-based rehabilitation alone. In accordance with the Strength of Recommendation Taxonomy, the grade of $\mathrm{A}$ is recommended based on consistent evidence from multiple high-quality RCTs that addressed the focused clinical question posed in this CAT.

\section{Implications for Practice, Education, and Future Research}

The purpose of this CAT was to critically appraise and synthesize the currently available evidence which compared interventions that included manual therapy in addition to exercise-based rehabilitation to exercise-based rehabilitation alone in patients with CAI. The results revealed consistent high-quality patient-oriented evidence demonstrating a greater effect when manual therapy was included with an exercise-based rehabilitation on PRO scores in patients with CAI. Burcal et $\mathrm{al}^{9}$ did not identify statistically significant differences between interventions; however, effect sizes supported the intervention group that coupled manual therapy with balance training. The conclusions reached by this study were likely impacted by a small sample size. However, Plaza-Manzano et $\mathrm{al}^{10}$ and Shih et al ${ }^{11}$ were also high-quality studies which provided support that manual therapy enhanced the outcomes of exercise-based rehabilitation interventions in people with CAI. Therefore, currently available interventions suggest that there is likely benefit to including manual therapy with exercise-based rehabilitation when designing treatment plans for patients with CAI.

The 2 studies ${ }^{10,11}$ that clearly demonstrated greater improvements in PRO scores used interventions that included manual therapy and multifaceted exercise-based rehabilitation programs. Their exercise-based rehabilitation intervention included either a proprioception and ankle strengthening or a neuromuscular training program. The greatest differences between groups were exhibited in the study by Plaza-Manzano et $\mathrm{al}^{10}$ that used a 4-week proprioceptive and strengthening rehabilitation program. The exercises incorporated in this program were static and dynamic single-limb balance tasks and eccentric strengthening of the ankle. The static balance tasks were single-limb balance with eyes open or closed. The dynamic tasks were single-limb balance while drawing a 5-point star, single-limb balance while catching a ball from the floor, and single-limb balance while throwing a ball. Each balance task progressed from a stable to an unstable surface throughout the 4-week program. The other exercises also increased in difficulty each week. Shih et $\mathrm{al}^{11}$ incorporated similar single-limb static and dynamic balance tasks into their rehabilitation protocol. However, these 2 studies differed in that Shih et al $^{11}$ did not incorporate eccentric strengthening or progress the difficulty of the exercises over the the 4 weeks of the study. This lack of variation in the exercises may not have allowed for as great of improvement in this group; thus, their PRO scores did not demonstrate as large of improvements. Burcal et $\mathrm{al}^{9}$ used a well-established dynamic balance training program as the exercise-based rehabilitation component. This balance training program has consistently demonstrated the ability to create large magnitude improvements in PRO scores in those with CAI, suggesting that other interventions may not enhance patient-reported function when coupled with this intervention. However, it is possible that a larger sample size may have yielded conclusions in support of the combined manual therapy and balance training intervention based on the effect sizes observed in this study. Therefore, manual therapy may improve patient outcomes when coupled with a diverse range of exercisebased rehabilitation interventions.

In addition to differences in the rehabilitation interventions between studies, the manual therapy interventions also varied across studies. All 3 studies ${ }^{9-11}$ incorporated nonweight-bearing mobilizations of the talocrural joint, but each described variations in the technique, duration, and number of mobilizations per treatment session. Plaza-Manzano et al ${ }^{10}$ incorporated talocrural joint mobilizations in the directions of traction, anteroposterior, posteroanterior, in addition to anteroposterior and posteroanterior distal tibiofibular joint mobilizations. This study also incorporated superficial peroneal nerve neurodynamic mobilization, which was not incorporated by the included studies. All mobilizations were grade III Maitland mobilizations, and all manual therapies were performed 10 times by 2 physiotherapists for a duration of 20 to 30 seconds with a 2-minute rest between techniques. Shih et al ${ }^{11}$ performed 5 minutes of end-range intermittent talocrural traction, 2 minutes of grade III and 2 minutes of grade IV anteroposterior talocrural mobilizations, and 2 minutes of grade III and 2 minutes of grade IV distal fibular mobilizations. Burcal et al used talocrural traction described as "mild and constant" and grade III Maitland ankle joint mobilizations. In addition, Burcal et $\mathrm{al}^{9}$ incorporated plantar massage and calf stretching in conjunction with joint mobilization in their treatment intervention for a total of 5 minutes of manual therapy treatment each session. The manual therapy technique used in the Plaza-Manzano et $\mathrm{al}^{10}$ study resulted in the greatest within-group as well as betweengroup differences in their respective PROs. The amount and duration of joint mobilizations in each participant received was greatest in this study, which could be a factor that contributed to the more significant results. These variations in manual therapy techniques should be controlled for in future research to establish a recommended standard of care for application of joint mobilizations in patients with CAI.

In addition to variations in manual therapy technique, these studies also differed in the PRO they used. Both Plaza-Manzano et $\mathrm{al}^{10}$ and Shih et $\mathrm{al}^{11}$ included a battery of PROs including the Cumberland Ankle Instability Tool, visual analog scale, and Global Rating Scale, whereas Burcal et $\mathrm{al}^{9}$ used the Foot and Ankle Ability 
Measure (FAAM) and the Foot and Ankle Ability Measure-Sport (FAAM-S). ${ }^{12}$ These outcome tools are not intended for the same purpose, and their data should not be weighed equally. The CAIT ${ }^{13}$ was designed as a discriminative tool that is typically used to identify individuals with CAI, whereas the FAAM and FAAM-S are evaluative tools developed to measure the patient's perceived level of function. ${ }^{12}$ Both Plaza-Manzano et $\mathrm{al}^{10}$ and Burcal et $\mathrm{al}^{9}$ provided effect sizes for their studies. The Cohen $d$ effect sizes for both the VAS and the CAIT in the Plaza-Manzano et $\mathrm{al}^{10}$ study were strong, 1.23 and 1.45 , respectively. The Hedges $g$ effect sizes for the FAAM and FAAM-S in the Burcal et $\mathrm{al}^{9}$ study were weak at -0.25 and -0.08 , respectively. Both Cohen $d$ and Hedges $g$ are interpreted using the same scale; 0.2 to 0.4 are small effect sizes, 0.5 to 0.7 are medium effect sizes, and 0.8 or greater are large effect sizes. We calculated the effect sizes for Shih et $\mathrm{al}^{11}$ and found that there was a moderate effect size (0.69) between the manual therapy group and the training group and a strong effect size (1.36) between the manual therapy group and the control group.

Two of 3 studies $^{10,11}$ agreed that incorporating joint mobilizations in addition to exercise-based rehabilitation improves PROs in CAI individuals. While Burcal et $\mathrm{al}^{9}$ indicated that there was no statistical difference between interventions, effect sizes supported the success of the intervention which included manual therapy. Therefore, in the context of this CAT, there is sufficient evidence to support the benefit of coupling joint mobilizations with exercise-based rehabilitation protocols to improve PROs. Future studies should further examine the long-term differences in outcomes between participants who complete exercise-based rehabilitation versus those who receive manual therapy in conjunction with exercise-based rehabilitation in order to support the use of joint mobilizations as a viable intervention for those with CAI. Plaza-Manzano et al ${ }^{10}$ identified better outcomes for several measures at 1-month follow-up indicating manual therapies may generate superior long-term effectiveness; however, additional studies with longitudinal data capture are needed. Further studies should also investigate other PROs, such as the Fear-Avoidance Beliefs Questionnaire, ${ }^{14}$ the Tampa Scale of Kinesiophobia, ${ }^{15}$ or the Disablement in the Physically Active Scale ${ }^{16}$ to investigate other important aspects of HRQOL which are impacted in patients with CAI. Examining the long-term effects of manual therapy on these PROs could influence the management of patients with CAI and influence the overall HRQOL of these patients. The clinical bottom line of this CAT was based on 3 high-quality RCTs. This recommendation should be revisited if additional best evidence becomes available within the scope of the focused clinical question.

\section{References}

1. Hootman JM, Dick R, Agel J. Epidemiology of collegiate injuries for 15 sports: summary and recommendations for injury prevention initiatives. J Athl Train. 2007;42(2):311-319. PudMed ID: 17710181

2. Feger MA, Glaviano NR, Donovan L, et al. Current trends in the management of lateral ankle sprain in the United States. Clin J Sport Med. 2017;27(2):145-152. PudMed ID: 27347860 doi:10.1097/JSM. 0000000000000321
3. Anandacoomarasamy A, Barnsley L. Long term outcomes of inversion ankle injuries. Br J Sports Med. 2005;39(3):e14. PudMed ID: 15728682 doi:10.1136/bjsm.2004.011676

4. Gribble PA, Delahunt E, Bleakley CM, et al. Selection criteria for patients with chronic ankle instability in controlled research: a position statement of the International Ankle Consortium. $J$ Athl Train. 2013;49(1):121-127. PudMed ID: 24377963 doi:10.4085/ 1062-6050-49.1.14

5. Houston MN, Van Lunen BL, Hoch MC. Health-related quality of life in individuals with chronic ankle instability. J Athl Train. 2014;49(6): 758-763. PudMed ID: 25299444 doi:10.4085/1062-6050-49.3.54

6. Kosik KB, Gribble PA. The effect of joint mobilization on dynamic postural control in patients with chronic ankle instability: a critically appraised topic. J Sport Rehabil. 2018;27(1):103-108. PudMed ID: 27992241 doi:10.1123/jsr.2016-0074

7. Hoch MC, Andreatta RD, Mullineaux DR, et al. Two-week joint mobilization intervention improves self-reported function, range of motion, and dynamic balance in those with chronic ankle instability. $J$ Orthop Res. 2012;30(11):1798-1804. PudMed ID: 22610971 doi:10. 1002/jor.22150

8. Powden CJ, Hoch JM, Hoch MC. Rehabilitation and improvement of health-related quality-of-life detriments in individuals with chronic ankle instability: a meta-analysis. J Athl Train. 2017;52(8):753-765. PudMed ID: 28704635 doi:10.4085/1062-6050-52.5.01

9. Burcal CJ, Trier AY, Wikstrom EA. Balance training versus balance training with STARS in patients with chronic ankle instability: a randomized controlled trial. J Sport Rehabil. 2017;26(5):347-357. PudMed ID: 27632839 doi:10.1123/jsr.2016-0018

10. Plaza-Manzano G, Vergara-Vila M, Val-Otero S, et al. Manual therapy in joint and nerve structures combined with exercises in the treatment of recurrent ankle sprains: a randomized, controlled trial. Man Ther. 2016;26:141-149. PudMed ID: 27598553 doi:10.1016/j. math.2016.08.006

11. Shih Y-F, Yu H-T, Chen W-Y, Liao K-K, Lin H-C, Yang Y-R. The effect of additional joint mobilization on neuromuscular performance in individuals with functional ankle instability. Phys Ther Sport. 2018; 30:22-28. PudMed ID: 29310055 doi:10.1016/j.ptsp.2017.12.001

12. Carcia CA, Martin RL, Drouin JM. Validity of the foot and ankle ability measure in athletes with chronic ankle instability. J Athl Train. 2008;43(2):179-183. PudMed ID: 18345343 doi:10.4085/10626050-43.2.179

13. Hiller CE, Refshauge KM, Bundy AC, Herbert RD, Kilbreath SL. The Cumberland ankle instability tool: a report of validity and reliability testing. Arch Phys Med Rehabil. 2006;87(9):1235-1241. PudMed ID: 16935061 doi:10.1016/j.apmr.2006.05.022

14. Waddell G, Newton M, Henderson I, Somerville D, Main CJ. A Fear-Avoidance Beliefs Questionnaire (FABQ) and the role of fearavoidance beliefs in chronic low back pain and disability. Pain. 1993;52(2):157-168. PudMed ID: 8455963 doi:10.1016/0304-3959 (93)90127-B

15. Weermeijer JD, Meulders A. Clinimetrics: Tampa Scale for kinesiophobia. J Physiother. 2018;64(2):126. PudMed ID: 29567379 doi:10. 1016/j.jphys.2018.01.001

16. Vela LI, Denegar CR. The disablement in the physically active scale, part II: the psychometric properties of an outcomes scale for musculoskeletal injuries. J Athl Train. 2010;45(6):630-641. PudMed ID: 21062187 doi:10.4085/1062-6050-45.6.630 